J. Austral. Math. Soc. 21 (Series A) (1976), 5-11.

\title{
TOTALLY PURE SEQUENCES AND TOTALLY PURE PROJECTION
}

\author{
DONALD COOK \\ (Received 6 November 1972) \\ Communicated by G. E. Wall
}

\section{Introduction}

All groups in this paper are Abelian. The notation is similar to Fuchs (1970) and Maclane (1963).

A reduced group $G$ is totally projective if $G / p^{\alpha} G$ is $p^{\alpha}$-projective for all ordinals $\alpha$. Nunke (1963) introduced this class of groups and derived a number of their properties. This class is of great interest since Hill (1967) has shown that Ulm's theorem holds for it. Nunke introduced the class via his study of $p^{x}$-purity, and his derivation of the various properties of totally projective groups depends heavily on the rather extensive theory described in Nunke (1963) and Nunke (1967). Crowley and Hales (1969) then introduced the concept of $T$-groups and proved Ulm's theorem for this class. Using Hill's theorem they showed that the class of $T$-groups coincides with the class of totally projective groups.

However, neither paper explicitly describes the elements of these groups. We shall investigate the relative homological algebra for which the totally projectives are projective. This will enable us to explicitly describe this class.

\section{The $\alpha$-Totally Pure Sequences}

Definition 1.1. Let $E: 0 \rightarrow A \rightarrow B \rightarrow C \rightarrow 0$ be exact. Then $E$ is $\alpha$-totally pure if and only if the induced sequences $p^{\beta} B[p] \rightarrow p^{\beta} C[p] \rightarrow 0$ are exact for each ordinal $\beta<\alpha$. If $E$ is $\alpha$-totally pure for each ordinal $\alpha$, then $E$ is totally pure.

Recall that the length of a $p$-group $G$ is the least ordinal $\alpha$ such that $p^{x} G=0$ and is denoted by $\Lambda(G)$.

THEOREM 1.1. Let $E: 0 \rightarrow A \rightarrow B \rightarrow C \rightarrow 0$ be exact. If $\alpha$ is a limit ordinal or $\Lambda(C) \leqq \alpha$ the following are equivalent.

(i) $E$ is $\alpha$-totally pure 
(ii) $0 \rightarrow p^{\beta} A[p] \rightarrow p^{\beta} B[p] \rightarrow p^{\beta} C[p] \rightarrow 0$ is exact for $\beta<\alpha$

(iii) $0 \rightarrow p^{\beta} A \rightarrow p^{\beta} B \rightarrow p^{\beta} C \rightarrow 0$ is exact for $\beta<\alpha$

(iv) $0 \rightarrow A / p^{\beta} A \rightarrow B / p^{\beta} B \rightarrow C / p^{\beta} C \rightarrow 0$ is exact for $\beta<\alpha$

(v) $0 \rightarrow p^{\lambda} A[p] / p^{\beta} A[p] \rightarrow p^{\lambda} B[p] / p^{\beta} B[p] \rightarrow p^{\lambda} C[p] / p^{\beta} C[p] \rightarrow 0$ is exact for $i<\beta<\alpha$.

Proof. For any ordinal $\alpha$, (i) is equivalent to (ii). If $\alpha$ is a limit ordinal, then (ii) implies (iii) easily. By using the $3 \times 3$ Lemma, (iii), (iv), (v) and (i) are equivalent.

If $\alpha=\lambda+n$ and $\Lambda(C)=\alpha$ with $\lambda$ a limit ordinal and $n<\omega$ then $p^{\lambda} C$ is bounded. Thus the sequence $0 \rightarrow p^{i} A \rightarrow p^{\lambda} B \rightarrow p^{i} C \rightarrow 0$ is split and the five conditions are equivalent for each ordinal $\beta<\alpha$.

COROLlary 1.1. Let $F_{6}(\alpha)$ denote the $\alpha$-th Ulm invariant of $G$. Let $E: 0 \rightarrow A \rightarrow B \rightarrow C \rightarrow 0$ be totally pure. Then $F_{B}(\alpha)=F_{A}(\alpha)+F_{C}(\alpha)$ for each ordinal $\alpha$.

Proof. By Condition (v) of Theorem 1.1, if $E$ is totally pure then

$$
0 \rightarrow p^{\alpha} A[p] / p^{\alpha+1} A[p] \rightarrow p^{\alpha} B[p] / p^{\alpha+1} B[p] \rightarrow p^{\alpha} C[p] / p^{\alpha+1} C[p] \rightarrow 0
$$

is exact. Over the $p$ element field this sequence splits. Hence $F_{B}(\alpha)=F_{A}(\alpha)+F_{C}(\alpha)$.

All split exact sequences are totally pure. There are totally pure sequences that are not split exact. Let $G$ be a group with $p^{\omega} G=0$ and $G$ is not a direct sum cyclics. Then there is a totally pure sequence $E: 0 \rightarrow A \rightarrow B \rightarrow C \rightarrow 0$ with $B$ a direct sum of cyclic groups. Hence $E$ does not split.

For any group $G$, let $G^{*}$ be the completion of $G / p \omega G$ in the $p$-adiac topology.

Then $E: 0 \rightarrow A \rightarrow B \rightarrow C \rightarrow 0$ is $(\omega+1)$-totally pure if and only if $0 \rightarrow A^{*} \rightarrow B^{*}$ $\rightarrow C^{*} \rightarrow 0$ splits (Cook (to appear)). Thus if $\alpha$ is not a limit ordinal, Theorem 1.1 need not hold. If the conditions of Theorem 1.1 are fulfilled then $A$ is an $\alpha$-isotype subgroup of $B$, Irwin, Walker and Walker (1963). The converse need not hold. If $p^{\omega} G=0$, then a basic subgroup is an $(\omega+1)$-isotype subgroup, but not a $(\omega+1)$-totally pure subgroup.

Definition 1.2. For each ordinal $\alpha$, let $P \operatorname{ext}_{\alpha}(C, A)$ be the subgroup of $\operatorname{Ext}(C, A)$ consisting of equivalence classes of $\alpha$-totally pure sequences.

THEOREM 1.2. If $\alpha$ is a limit ordinal, then $A \subseteq p^{\alpha} B$ if and only if the sequences

$$
\begin{aligned}
E_{D}: 0 \rightarrow \operatorname{Hom}(D, A) & \rightarrow \operatorname{Hom}(D, B) \rightarrow \operatorname{Hom}(D, B / A) \rightarrow \\
\operatorname{Ext}(D, A) & \rightarrow \operatorname{Pext}_{x}(D . B) \rightarrow \operatorname{Pext}_{x}(D, B / A)
\end{aligned}
$$

are exact for all divisible groups $D$.

Proof. Suppose $E_{D}$ is exact for all divisible groups $D$. Let $A \subset D$ with $D$ divisible. Then under the map $\operatorname{Ext}(D / A, A) \rightarrow P \operatorname{ext}_{z}(D / A . B)$ the element rep- 
resented by $0 \rightarrow A \rightarrow D \rightarrow D / A \rightarrow 0$ maps onto the element represented by $0 \rightarrow B \stackrel{f}{\rightarrow} D \oplus B / M \stackrel{g}{\rightarrow} D / A \rightarrow 0$ where $M=\{(a,-a) \mid a \in A\}, \quad f(x)=(x, 0)$ $+M$ and $g((x, d)+M)=d+A$ for $x \in B$ and $d \in D$. Let $a \in A \subset D$. If $\beta<\alpha$, then $a \in p^{\beta} D$ implies

$$
f(a)=(a, 0)+M=(0, a)+M \in p^{\beta}(B \oplus D / M) .
$$

Since $g((0, a)+M)=0$ and $0 \rightarrow p^{\beta} B \rightarrow p^{\beta}(B \oplus D / M) \rightarrow D / A \rightarrow 0$ is exact, there is $b \in p^{\beta} B$ such that $f(b)=(0, a)+M=f(a)$. Since $f$ is a monomorphism $a=b$ and $a \in p^{\beta} B$ for $\beta<\alpha$. Hence, $a \in p^{\beta} B$ for each $\beta<\alpha$. Thus $A \subset p^{\alpha} B$.

If $A \subset p^{\beta} B$, then $E_{D}$ is exact (Nunke (1963)).

COROLlaRY 1.2. Let $\alpha$ be an ordinal. If $C$ is $\alpha$-totally pure projective, then $\operatorname{Ext}(C, A) \cong \operatorname{Ext}\left(C, A / p^{\alpha} A\right)$ for each group $A$.

Proof. For $p^{\alpha} A$, the sequence $\operatorname{Hom}\left(C, A / p^{\alpha} A\right) \rightarrow \operatorname{Ext}\left(C, p^{\alpha} A\right) \rightarrow P \operatorname{ext}_{\alpha}(C, A)$, $=0$ is exact. Since the sequence $\operatorname{Ext}\left(C, p^{\alpha} A\right) \rightarrow \operatorname{Ext}(C, A) \rightarrow \operatorname{Ext}\left(C, A / p^{\alpha} A\right) \rightarrow 0$ is exact, then $\operatorname{Ext}(C, A) \cong \operatorname{Ext}\left(C, A / p^{\alpha} A\right)$.

\section{The $\alpha$-Totally Pure Projectives}

To describe the $p$-groups that are $\alpha$-totally pure projectives, Nunke (1967) introduces a class of groups, one for each ordinal $\alpha$. These groups are defined homologically and their basic properties are derived by the same means. Crowley and Hales (1969), define the concept of $T$-groups and using Hill's theorem show that these two classes coincide. In our development, we introduce similar classes of groups, but its definitions and basic properties will be in terms of the most elementary group theoretic principles. Via this class of groups, we will describe the $\alpha$-totally pure projectives, derive some of their basic properties, and show that there are enough of them.

Definition 2.1. (Crowley and Hales) Let $X$ be a set and $V \subset X^{2}$, let $u$ and $v$ be maps of $X$ and $V$ into the nonnegative integers respectively. Let $G(X, V, u, v)$ be the Abelian group generated by $X$ subject only to the relations

$$
\begin{aligned}
& p^{u(x)}=0 \text { for all } x \in X \\
& p^{p(x . y)}=y \text { for all }(x, y) \in V .
\end{aligned}
$$

Then an Abelian p-group $G$ is a $T$-group if $G \simeq G(X, V, u, v)$ for some quadruple $(X, V, u, v)$.

Definition 2.2. Let $\alpha$ be an ordinal, $X_{\alpha}=\left\{\alpha_{1} \alpha_{2} \cdots \alpha_{n} \mid \alpha_{i}\right.$ an ordinal, $\left.\alpha>\alpha_{1}>\alpha_{2} \cdots \alpha_{n}\right\} ; F_{\alpha}$ the free group on $X_{\alpha} ; K_{\alpha}$ the subgroup of $F_{\alpha}$ generated by the elements $\left\{p \alpha_{1} \alpha_{2} \cdots \alpha_{n+1}-\alpha_{1} \alpha_{2} \cdots \alpha_{n}, p \beta\right\}$; and $P_{x}=F_{x} / K_{x}$. 
The element $\alpha_{1} \alpha_{2} \cdots \alpha_{n}+K_{\alpha}$ in $P_{\alpha}$ will be simply denoted by $\alpha_{1} \alpha_{2} \cdots \alpha_{n}$. It is clear that $P_{\alpha}$ is a $T$-group. Moreover $p^{n} \alpha_{1} \alpha_{2} \cdots \alpha_{n}=0$.

To motivate this definition suppose that $E: 0 \rightarrow A \rightarrow B \rightarrow C \rightarrow 0$ is totally pure. If $c \in p^{a} C$ then $=p c_{\alpha_{1}}$ with $c_{\alpha_{1}} \in p^{\alpha_{1}} C$ and $\alpha>\alpha_{1}$. Now $c_{\alpha_{1}}=p c_{\alpha_{2}}$ with $c_{\alpha_{2}} \in p^{\alpha_{2}} C$ and $\alpha>\alpha_{1}>\alpha_{2}$. Since every decreasing sequence of ordinals is finite we obtain a set $c, c_{\alpha_{1}}, c_{\alpha_{2}} \cdots c_{\alpha_{n}}$ subject to certain relations. Since $E$ is totally pure we can lift each $c_{\alpha_{i}}$ to $b_{\alpha_{i}} \in p^{\alpha_{i}} B$ and the set $b_{\alpha}, b_{\alpha_{i}} \cdots b_{\alpha_{n}}$ is also subject to these relations. What we have done is to construct a class with only these relations. This class should be, and indeed is, the class of totally pure projectives. We first define some important subgroups of the $P_{\alpha}$ 's.

Definition 2.3. Let $H_{\alpha}=\left\langle\left\{\alpha \alpha_{1} \cdots \alpha_{n} \in P_{\alpha+1}|\alpha\rangle \alpha_{1} \cdots \alpha_{n}\right\}\right\rangle$

The next three propositions may be proven by transfinite induction, or they follow directly from statements (3.3), (3.4) and (3.5) in Crowley and Hales (1969).

Proposition 2.1. Let $\alpha$ be an ordinal. Then $P_{\alpha}=\Sigma_{\beta<\alpha} H_{\beta}$.

Proposition 2.2. Each element $y \in P_{\alpha}$ can be written uniquely in the form $y=\Sigma_{x \in X_{x}} s_{x} s, 0 \leqq s_{x}<p$.

Proposition 2.3. Let $\gamma$ be an ordinal and $X_{z}^{\gamma}=\left\{\alpha_{1} \alpha_{2} \cdots \alpha_{n} \mid \alpha_{n} \geqq \gamma\right\}$. Then $p^{\gamma} P_{\alpha}$ is the subgroup generated by $X_{\alpha}^{\gamma}$.

In view of Proposition 2.3, it can be noted that the height of the ordinal $\beta$ in $P_{\alpha}$ is precisely $\beta$.

The next theorem starts to connect the $H_{\alpha}$ 's with the $\alpha$-totally pure sequences.

TheORem 2.1. For $c \in C, c \in p^{\alpha} C[p]$ if and only if there is $f \in \operatorname{Hom}\left(H_{x}, C\right)$ such that $f(\alpha)=c$.

Proof. If $f \in \operatorname{Hom}\left(H_{\alpha}, C\right)$, then $f(\alpha) \in p^{\alpha} C[p]$. Suppose $c \in p^{\alpha} C[p]$. We will define a set map

$$
f: H_{\alpha} \cap X_{\alpha} \rightarrow C \text { such that }
$$

(i) $f(\alpha)=c$

(ii) $f\left(\alpha \alpha_{1} \cdots \alpha_{n}\right) \in p^{\alpha_{n}} C\left[p^{n+1}\right]$

(iii) $p f\left(\alpha \alpha_{1} \cdots \alpha_{n}\right)=f\left(p \alpha \alpha_{1} \cdots \alpha_{n}\right)$.

Let $f(\alpha)=c \in p^{\alpha} C[p]$. Suppose for all $k \leqq n-1, f\left(\alpha \alpha_{1} \cdots \alpha_{k}\right)$ is defined st: $\cdot 1$ that $f\left(\alpha \alpha_{1} \cdots \alpha_{k}\right) \in p^{\alpha_{k}} C\left[p^{k+1}\right]$. Given $\alpha \alpha_{1} \cdots \alpha_{n}$, then $f\left(\alpha \alpha_{1} \cdots \alpha_{n+1}\right) \in p^{\alpha_{n}} C\left[p^{n}\right]$. Since $\alpha_{n-1}>\alpha_{n}$, then there is a $c_{\alpha_{n}} \in p^{x_{n}}\left[p^{n+1}\right]$ such that $p c_{\alpha_{n}}=f\left(\alpha \alpha_{1} \cdots_{-1}\right)$. Let $f\left(\alpha \alpha_{1} \cdots \alpha_{n}\right)=c_{\alpha n}$. Then $p f\left(\alpha \alpha_{1} \cdots \alpha_{n}\right)=p c_{\alpha_{n}}=f\left(p \alpha \alpha_{1} \cdots \alpha_{n}\right)$. Thus b, inite induction, $f$ is defined on all the generating elements of $H_{x}$. Since the rel in' 
among these elements are preserved and $f$ is a height increasing, order decreasing set map, then $f$ extends to a group homomorphism from $H_{\alpha}$ to $C$.

The next theorem characterizes the $\alpha$-totally pure sequences in terms of the $H_{\beta}$ 's.

THEOREM 2.2. The sequence $E: 0 \rightarrow A \rightarrow B \stackrel{\sigma}{\rightarrow} C \rightarrow 0$ is $\alpha$-totally pure if and only if $H_{\beta}$ is projective for all $\beta<\alpha$.

Proof. Suppose $H_{\beta}$ is projective for all $\beta<\alpha$ and $c \in p^{\beta} C[p]$. Then by Theorem 2.1 there is a homomorphism $f: H_{\beta} \rightarrow C$ such that $f(\beta)=c$. Since $H_{\beta}$ is projective there is a homomorphism $g: H_{\beta} \rightarrow B$ with $\sigma g=f$. Thus $\sigma_{\beta} g(\beta)=c$ and $p^{\beta} B[p] \rightarrow 0$ is exact.

Suppose $E$ is $\alpha$-totally projective. If $P_{\beta}$ is projective for $\beta \leqq \alpha$, then $H_{\beta}$ is projective for $\beta<\alpha$. Thus suppose for all $\gamma<\beta, P_{\gamma}$ is projective. If $\gamma$ is a limit ordinal, then $P_{\gamma}=\Sigma_{\beta<\gamma} H_{\beta}$ and $P_{\gamma}$ is projective. If $\gamma=\beta+1$, then $P_{\gamma}=H_{\beta} \oplus P_{\beta}$. Hence it suffices to show $H_{\beta}$ is projective. Suppose $f: H_{\beta} \rightarrow C$. Then $f(\beta) \in p^{\beta} C[p]$ and there is $b \in p^{\beta} B[p]$ with $\sigma(b)=f(\beta)$. Thus $b=\rho(\beta)$ for some $\rho: H_{\beta} \rightarrow B$. Since $(f-\sigma \rho)(\beta)=0$ and $H_{\beta} /<\beta>=P_{\beta}$, then $f-\sigma \rho=\lambda \pi$ where $\pi: H_{\beta} \rightarrow P_{\beta}$. Since $P_{\beta}$ is projective $\lambda=\sigma \delta$ so that $f=\sigma(\rho+\delta \pi)$. Thus $P_{\gamma}$ is projective. Hence for $\beta<\alpha, H_{\beta}$ is projective.

The reader is refered, to Maclane (1963) for a proof of the following corollary.

COROLlaRY 2.1. The $\alpha$-totally pure sequences are a proper class.

COROLlary 2.2. The sequence $0 \rightarrow A \rightarrow B \rightarrow C \rightarrow 0$ is $\alpha$-totally pure if and only if the sequence of p-primary subgroups, $0 \rightarrow A_{p} \rightarrow B_{p} \rightarrow C_{p} \rightarrow 0$, is $\alpha$-totally pure.

Proor. This follows readily since $\operatorname{Hom}\left(H_{\beta}, G_{p}\right)=\operatorname{Hom}\left(H_{\beta}, G\right)$ for each $H_{\beta}$.

THEOREM 2.3. Let $\alpha$ be an ordinal and $G$ a group. Then there is a sequence $E: 0 \rightarrow K \rightarrow P \rightarrow G \rightarrow 0$ with $P \alpha$-totally projective and $E \alpha$-totally pure.

Proof. Let $P=F \oplus T$ where $F$ is free and $F \rightarrow G \rightarrow 0$ is exact, and $T=\sum_{\beta<\alpha} \sum_{f \in \operatorname{Hom}\left(H_{\beta}, G\right)} H_{\beta}$.

If $G$ is a reduced $p$-group and $\Lambda(G)=\beta$ and $\alpha \geqq \beta$, then any $\alpha$-totally pure sequence, $E: 0 \rightarrow K \rightarrow P \rightarrow G \rightarrow 0$ is totally pure. If $P$ is $\alpha$-totally pure projective, 'h $P$ is totally pure projective. Hence if $G$ is a reduced $p$-group, then there is a coilly pure projective $P$ and epimorphism $f: P \rightarrow G$ with $\operatorname{Ker} f$ totally pure in $P$. The next theorem shows that if $G$ is a divisible $p$-group, then $G$ is totally e p-ojective.

OREM - 4 . The group $Z\left(p^{\infty}\right)$ is totally pure projective. 
Proof. Suppose $E: 0 \rightarrow A \rightarrow B \rightarrow C \rightarrow 0$ is totally pure. Then there is an ordinal $\alpha$ so that $p^{\alpha} A, p^{\alpha} B$, and $p^{\alpha} C$ are divisible. Since the sequence $0 \rightarrow p^{\alpha} A$ $\rightarrow p^{\alpha} B \rightarrow p^{\alpha} C \rightarrow 0$ splits, $Z\left(p^{\infty}\right)$ is totally pure projective.

Combining Theorems 2.3 and 2.4 we hàve the following theorem.

THEOREM 2.5. Let $G$ be a group. Then there is a totally pure sequence $E: 0 \rightarrow K \rightarrow P \rightarrow G \rightarrow 0$ with $P$ totally pure projective.

THEOREM 2.6. Let $G$ be a reduced p-group. If $G$ is $\alpha$-totally pure projective, then $G$ is a direct summand of a direct sum of $H_{\beta}$ 's for $\beta<\alpha$. Hence $\Lambda(G) \leqq \alpha$.

Proof. Let $E: 0 \rightarrow K \rightarrow P \rightarrow G \rightarrow 0$ be $\alpha$-totally pure with $P$ as in Theorem 2.3. Then $E$ splits and $G$ is a summand of $P$. Since $G$ is a $p$-group and summands of free groups are free, then $G$ is a summand of $H_{\beta}$ for $\beta<\alpha$.

Recall that the set $X_{\beta}^{\gamma}=\left\{\beta \alpha_{1} \cdots \alpha_{n} \in H_{\beta} \mid \alpha_{n} \geqq \gamma\right\}$. Then $H_{\beta} / p^{\gamma} H_{\beta}=\Sigma_{\Gamma \in X_{\beta}^{\gamma}}\left(P_{\gamma}\right)_{\Gamma}$. If $G$ is a $\alpha$-totally pure projective, then there is a group $H$ such that $G \oplus H=\Sigma H_{\beta}$ for $\beta$ 's less than $\alpha$. Then $p^{\gamma} G \oplus p^{\gamma} H=\sum p^{\gamma} H_{\beta}$ and $p^{\gamma} G$ is $\alpha$-totally projective. Since $G \approx H / p^{\beta} G \oplus p^{\beta} H \approx G / p^{\beta} G \oplus H / p^{\beta} H \approx \sum H_{\gamma} / p^{\beta} H_{\gamma} \approx \Sigma\left(\sum P_{\beta}\right), G / p_{\gamma} G$ is also $\alpha$-totally projective. Using the results of this section, one can show directly that if $p^{\gamma} G$ and $G / p^{\gamma} G$ are $\alpha$-totally projective, then so is $G$.

Nunke (1967) defines totally projective groups. These are the reduced totally pure projective groups.

The following theorem and proof is due to Professor Fred Richman.

THEOREM 2.7. $A$ p-group $G$ is totally injective if $G=T \oplus D$ with $T$ torsion complete and $D$ divisible.

Proof. Choose $B$, a direct sum of cyclics, such that $|G|^{|B|}<2^{|B|}$. Then the sequence $\operatorname{Hom}(B, G) \rightarrow P \operatorname{ext}_{\omega}(\bar{B} / B, G) \rightarrow P \operatorname{ext}_{\omega}(\bar{B}, G)$ is exact. Any pure extension by a group without elements of infinite height is totally pure, so $P \operatorname{ext}_{\omega}(\bar{B}, G)=0$. $|\operatorname{Hom}(B, G)| \leqq|G|^{|B|}<2^{|B|}$. So $P \operatorname{ext}_{\omega}(\bar{B} \mid B, G)=\Pi P \operatorname{ext}{ }_{\omega}\left(Z_{p \infty}, G\right)$ is smaller than $2^{|B|}$. Thus $P$ ext ${ }_{t o}\left(z_{p m}, G\right)=0$ and so $G$ is torsion ${ }^{|B|}$ complete $\oplus$ divisible.

\section{References}

D. Cook (to appear), 'Complete Exact Sequences', Fund. Math.

P. Crowley and A. W. Hales (1969), 'The structure of Abelian p-groups given by certain presentations', J. Algebra 12, 10-23.

L. Fuchs (1970), Infinite Abelian Groups, Vol. 1 (Academic Press, New York and London (1970)).

P. Hill (1967), 'Ulm's theorem for totally projective groups' Abstract 652-15, Notices Am. Soc. $14,940$.

J. M. Irwin, C. Walker and E. A. Walker (1963), On $p^{\alpha}$-pure sequences of Abelian groups, Topics in Abelian Groups (ed. by J. M. Irwin and E. A. Walker, Scott Foresman and Co., Chicago (1963), 69-119).

S. MacLane (1963), Homology (Springer-Verlag, Berlin (1963)). 
R. J. Nunke (1963), Purity and subfunctions of the identity, Topics in Abelian Groups, (ed. by J. M. Irwin and E. A. Walker, Scott-Foresman and Co., Chicago (1963)), 121-171.

R. J. Nunke (1967), 'Homology and direct sums of countable Abelian groups', Marh. $Z$. 101, 182-212.

F. Richman (to appear), 'Totally injectives are torsion complete plus divisible'.

H. Ulm (1933), 'Zur theorie der abzäh̆lbarunendlichen abelschen Gruppen', Math. Ann. 107, 774-803.

Kansas State University

Manhattan

Kansas 66502

U.S.A. 